\title{
Using mRNA deep sequencing to analyze differentially expressed genes during Panax notoginseng saponin treatment of ischemic stroke
}

\author{
JUN LIN, PING LIANG, QING HUANG, CHONGDONG JIAN, JIANMIN HUANG, XIONGLIN TANG, \\ XUEBIN LI, YANLING LIAO, XIAOHUA HUANG, WENHUA HUANG, LI SU And LANQING MENG \\ Department of Neurology, Affiliated Hospital of Youjiang Medical College for Nationalities, \\ Baise, Guangxi Zhuang Autonomous Region 533000, P.R. China
}

Received October 30, 2019; Accepted August 10, 2020

DOI: $10.3892 / \mathrm{mmr} .2020 .11550$

\begin{abstract}
Treatment with Panax notoginseng saponin (PNS) can prevent neurological damage in middle cerebral artery occlusion model rats to promote recovery after a stroke. However, the exact molecular mechanisms are unknown and require further study. In the present study, mRNA sequencing was employed to investigate differential gene expression between model and sham groups, and between model and PNS-treated groups. Enrichment of gene data was performed using Gene Ontology analysis and the Kyoto Encyclopedia of Genes and Genomes database. Hub genes were identified and networks were constructed using Cytoscape that were further verified by reverse transcription-quantitative PCR. A total of 1,104 genes of interest were found, which included 690 upregulated and 414 downregulated genes that were identified when the model was compared with the sham group. Additionally, 817 genes of interest, which included 390 upregulated and 427 downregulated genes, were identified when the PNS-treated group was compared with the model group. There were 303 overlapping genes of interest between the analysis of model to sham groups, and the analysis of model to PNS-treated groups. The top 10 genes from the 303 aberrantly expressed genes of interest included ubiquitin conjugating enzyme E2 variant 2, small ubiquitin-related modifier 1, small RNA binding exonuclease protection factor La, Finkel-Biskis-Reilly murine sarcoma virus (FBR-MuSV) ubiquitously expressed, centrosomal protein $290 \mathrm{kDa}$, DNA-directed RNA polymerase II subunit $\mathrm{K}$, cullin-4B, matrin-3 and vascular endothelial
\end{abstract}

Correspondence to: Dr Lanqing Meng, Department of Neurology, Affiliated Hospital of Youjiang Medical College for Nationalities, 18 Zhongshan Second Road, Baise, Guangxi Zhuang Autonomous Region 533000, P.R. China

E-mail: menglanqingbs@163.com

Key words: Panax notoginseng saponin, middle cerebral artery occlusion, ischemic stroke, mRNA deep sequencing growth factor receptor 2 . In conclusion, these genes may be important in the underlying mechanism of PNS treatment in ischemic stroke. Additionally, the present data provided novel insight into the pathogenesis of ischemic stroke.

\section{Introduction}

Stroke is the second leading cause of death and disability worldwide (1). Ischemic stroke, which accounts for $80 \%$ of all strokes, usually causes severe neuronal damage and secondary neuronal death (2). Cerebral ischemia and reperfusion injury are complex pathological processes that involve inflammation, apoptosis and neuronal stress that rely on progressive changes in protein expression $(3,4)$. Currently, there are very few effective drugs that can treat cerebral ischemia and reperfusion injury in the clinic (5). Medicinal plants, such as the popular traditional Chinese herb Panax notoginseng, also known as Sanqi, are often used to treat cerebrovascular and cardiovascular disorders in Traditional Chinese medicine (6). Panax notoginseng saponins (PNS) can be extracted from Sanqi, and its use can result in an effective neuroprotective effect and promote stroke recovery $(7,8)$. Compounds in the PNS mainly include ginsenoside Rb1, ginsenoside Rd, ginsenoside Rg1,notoginsenoside R1 and ginsenoside Re (9-11). A previous study found that ginsenoside Rb1 had anti-apoptotic, anti-oxidative and anti-inflammatory effects that ameliorated brain water content, promoted bioactivities associated with neurogenesis potential and had a neuroprotective effect (12). Ginsenoside Rd is known to attenuate ischemic stroke-induced damage through an anti-oxidative stress and anti-inflammatory response (13). Ginsenoside Rg1 treatment attenuated infarct volume and neurological function score, which demonstrated that it is a potential neuroprotective drug that could be used to treat ischemic stroke (14). A previous study found that PNS treatment attenuated oxygen-glucose deprivation/reoxygenation-induced injury in human SH-SY5Y cells (15). PNS treatment has also been demonstrated to inhibit the secretion of inflammatory factors, regulate Nogo-A, NgR and p75NGF expression, and to regulate the RhoA/ROCK2 pathway in permanent middle cerebral artery occlusion (MCAO) model rats to prevent neurological damage $(8,16,17)$. However, the 
molecular mechanisms underlying how PNS protects against ischemic injury remain unclear.

The purpose of the present study was to determine the gene expression profile in PNS-treated MCAO rats after ischemic stroke using mRNA deep sequencing, which may provide insight into the molecular mechanisms involved in PNS treatment of ischemic stroke.

\section{Materials and methods}

Treatments. PNS was purchased from Guangxi Wuzhou Pharmaceutical Group Co., Ltd., (China Food and Drug Administration approval no. Z20025652), which was mostly made up of ginsenoside Rb1 (29.7\%), ginsenoside Rd (7.3\%), ginsenoside $\operatorname{Rg} 1$ (28.0\%), notoginsenoside R1 (6.9\%) and ginsenoside $\operatorname{Re}(3.8 \%)$.

Establishing MCAO model and treatment conditions. The present study obtained approval from the Ethics Committee of Youjiang Medical College for Nationalities Institutional Review Board. A total of 60 male Sprague-Dawley (SD) rats of a specific pathogen-free grade (age, 7-weeks; weight, 200 $\pm 20 \mathrm{~g}$ ) were purchased from Changsha Tianqin Biotechnology Co. Ltd. [License Number: SCXK(Xiang)2014-0011]. SD rats were housed under 50-60\% humidity, 12/12 h light/darkness, and $20 \pm 2{ }^{\circ} \mathrm{C}$ room temperature with free access to drinking water and food. SD rats were randomly divided into three groups: Control group (rats without any treatment, $n=10$ ), Sham operation group (sham, $\mathrm{n}=10$ ) and MCAO group (model, $\mathrm{n}=40$ ). SD rats were fasted overnight, but allowed ad libitum access to water in the Youjiang Medical College [License Number: SYXK(Gui)2017-0004]. Rats were anesthetized for surgery by inhalation of isoflurane (4\% isoflurane for induction and $2 \%$ for maintenance). After $3 \mathrm{~min}$, SD rats were checked to ensure they were fully anesthetized, this was performed using the following methods: i) induction box was shaken gently, if the rat's body fell over to the side-lying position and it did not try to restore its prone position, it indicated that the animal was fully anesthetized; and ii) the toes of the rat were lightly clamped by tweezers, if the rat did not react after pinching, the rat was judged to be in a deep anesthesia. Then, MCAO rat models were established as previously described by Longa et al (18). Briefly, the common carotid artery (CCA), internal carotid artery (ICA) and external carotid artery (ECA) were carefully exposed after anesthesia. Then, the CCA and ECA were tied with a loose surgical knot. The ICA was tied with a loose knot with a silk suture. A small incision was made in the ECA and a silicon-coated monofilament was introduced through the CCA into the ICA $20 \mathrm{~mm}$ beyond the carotid bifurcation to occlude the MCA for $1 \mathrm{~h}$. Following surgery, the rats were housed individually and closely monitored for changes in behavior and vital signs. Neurological scores were assessed when rats awoke according to neurological deficit scores, where a score of 3-5 indicates that the model was successfully established (18). Then, MCAO rats were randomly divided into four groups: i) MCAO group; ii) $50 \mathrm{mg} / \mathrm{kg}$ PNS treatment group; iii) $100 \mathrm{mg} / \mathrm{kg}$ PNS treatment group; and iv) $150 \mathrm{mg} / \mathrm{kg}$ PNS treatment group. PNS treatment groups were given PNS by intragastric treatment once every 2 days. The rats in the control and sham groups were treated with the same volume of normal saline. Rats in the sham groups underwent an operation to expose the CCA, ICA and ECA after anesthesia but the ligation was not performed. Rats in the control group did not undergo surgery.

In order to minimize pain, the following characteristics were considered humane endpoints to the study that required immediate intervention: Infection at the surgical site, wound dehiscence, rapid weight loss ( $>20 \%$ body weight loss), becoming cachectic, self-harming, biting or aggressiveness, and difficulty eating, drinking or moving around freely. No rat was euthanized throughout the experiment. After treatment for 7 days, the rats were euthanized with pentobarbital (intraperitoneal injection, $120 \mathrm{mg} / \mathrm{kg}$ ). Cervical dislocation was used to confirm death.

Evaluation of neurological deficit and 2,3,5-triphenyltetrazolium chloride (TTC) staining. After treatment, the neurological deficit was evaluated by two researchers who were unaware of the groupings using a neurological deficit score as previously described $(18,19)$. Then, the extent and localization of the cerebral lesion were determined by TTC staining. Briefly, the brain was dissected in the coronal plane at the bifurcation of MCA and anterior cerebral artery, and the cerebral lesion was visualized by TTC staining. Briefly, 2-mm thick brain tissue sections were stained with $2 \%$ TTC solution at $37^{\circ} \mathrm{C}$ for $30 \mathrm{~min}$, followed by fixation with $4 \%$ paraformaldehyde. TTC-stained sections were photographed with a D5600 camera (Nikon). The digital images were analyzed using Image-Pro Plus 6.0 software (Media Cybernetics, Inc.).

$m R N A$ sequencing and differential gene analysis. Total RNA from sham, MCAO and $100 \mathrm{mg} / \mathrm{kg}$ PNS treatment groups was extracted using TRIzol ${ }^{\circledR}$ (Invitrogen; Thermo Fisher Scientific, Inc.). RNA concentration was measured using a NanoDrop ${ }^{\mathrm{TM}}$ 2000 spectrophotometer (Thermo Fisher Scientific, Inc.) at 260/280 nm and a Bioanalyzer 4200 (Agilent Technologies, Inc.). The libraries were prepared using a VAHTS mRNA-seq v2 Library Prep Kit for Illumina ${ }^{\circledR}$ (cat. no. NR601-01; Vazyme Biotech Co., Ltd.). The reverse transcription was conducted at $25^{\circ} \mathrm{C}$ for $10 \mathrm{~min}, 42^{\circ} \mathrm{C}$ for $15 \mathrm{~min}$, then $70^{\circ} \mathrm{C}$ for $15 \mathrm{~min}$. Adapters were ligated at the $3^{\prime}$ and $5^{\prime}$ ends, and templates were amplified using PCR. The PCR products were further purified using VAHTS ${ }^{\text {TM }}$ RNA Clean Beads (cat. no. N412-01; Vazyme Biotech Co., Ltd.) according to the manufacturer's protocol and used for sequencing. mRNAs were analyzed by deep sequencing using a HiSeq $X$ Ten Reagent kit v2.5 (cat. no. FC-501-2521; Illumina, Inc.) on an Illumina HiSeq $X$ sequencing platform (pair-ended; 150-bp reads; Illumina, Inc.).

The sequencing data was filtered using SOAPnuke (version 1.5.2), and clean reads were obtained after removing: i) reads containing sequencing adapters; ii) reads with low-quality base ratio $>20 \%$ (base quality $\leq 5$ ); and iii) reads with unknown base ( $\mathrm{N}$ base) ratio $>5 \%$. Differentially expressed genes were selected as having >two-fold differences between their geometrical mean expression in the compared groups, and a statistically significant P-value $(<0.05)$ by analysis of DEseq2. Gene Ontology (GO) analysis of differentially expressed genes was performed with an $\mathrm{R}$ package with cluster profiler using $\mathrm{P}<0.05$ to define statistically enriched GO categories (20-22). 
Table I. Primer sequences for reverse transcription-quantitative PCR.

\begin{tabular}{lll}
\hline Gene & \multicolumn{1}{c}{ Forward $\left(5^{\prime} \rightarrow 3^{\prime}\right)$} & \multicolumn{1}{c}{ Reverse $\left(5^{\prime} \rightarrow 3^{\prime}\right)$} \\
\hline UBE2V2 & GCGGTGCGTCTGGTAGAA & CGTGCATCCACCATTCCACT \\
SUMO1 & GGTGAATCCACCGACACCAT & CTCACTGCTGTCCTGTCCAA \\
SSB & GCTGGGTACCTTTGGAAACA & TATCATCAAGGGTGGCATCA \\
FAU & GCGGGTAAGTAGCCAACATG & AGCCTGCCAGAAGCACGAC \\
CEP290 & TCCAGTGGGCTACAGAGCAA & CTTTGTCAGGCTGTGGACCT \\
POLR2K & CCGGTAGTGTCTCTTGCTTC & CGAGCATCAAAAACCACCAATCT \\
CUL4B & AGTGACTCCGGCAAAAAGAGG & GGCGCTCTTGATTGGAGGTT \\
MATR3 & CCAAGAGGAAATCTGGGTGCT & ATGTGAACAACTCGGCTGGT \\
KDR & TCACGGTTGGGCTACTGC & AGACCTTCTGCCATCACG \\
PRPF40A & ACACTGGAGAGGAACCACCT & CATTGGAGGGTACCCGCTTT
\end{tabular}

UBE2V2, ubiquitin conjugating enzyme E2 variant 2; SUMO1, small ubiquitin-related modifier 1; SSB, small RNA binding exonuclease protection factor La; FAU, Finkel-Biskis-Reilly murine sarcoma virus ubiquitously expressed; CEP290, centrosomal protein $290 \mathrm{kDa}$; POLR2K, DNA-directed RNA polymerase II subunit K; CUL4B, cullin-4B; MATR3, matrin-3; KDR, vascular endothelial growth factor receptor 2; PRPF40A, pre-mRNA-processing factor 40 homolog A.

Pathway analysis was used to determine the significant pathway of the differential genes according to Kyoto Encyclopedia of Genes and Genomes (KEGG) database (http://www.genome. $\mathrm{jp} / \mathrm{kegg} /$ ) (23-25). Protein-protein interactions (PPI) were analyzed with the Search Tool for the Retrieval of Interacting Genes/Proteins (STRING; http://string-db.org) (26). Hub genes were analyzed by CytoHubba app in Cytoscape (27).

Reverse transcription-quantitative PCR (RT-qPCR). cDNA synthesis was carried out using PrimeScript ${ }^{\mathrm{TM}}$ RT reagent kit (Takara Biotechnology Co., Ltd.). The conditions of reverse transcription were as follows: $30^{\circ} \mathrm{C}$ for $10 \mathrm{~min}$ and $42^{\circ} \mathrm{C}$ for $60 \mathrm{~min}$, followed by $85^{\circ} \mathrm{C}$ for $10 \mathrm{~min}$. RT-qPCR was performed using the SYBR ${ }^{\circledR}$ Premix ExTaq ${ }^{\mathrm{TM}}$ II kit (Takara Biotechnology Co., Ltd.) on a 7500 Real-Time PCR System (Applied Biosystems; Thermo Fisher Scientific, Inc.). The RT-qPCR thermocycling conditions consisted of an initial denaturation at $95^{\circ} \mathrm{C}$ for $10 \mathrm{~min}$, followed by 40 cycles at $95^{\circ} \mathrm{C}$ for $15 \mathrm{sec}$ and $65^{\circ} \mathrm{C}$ for $32 \mathrm{sec}$. The relative mRNA expression levels were calculated using the $2^{-\Delta \Delta \mathrm{Cq}} \operatorname{method}(28)$. Glyceraldehyde 3-phosphate dehydrogenase (GAPDH) was used as the internal reference gene. The sequences of primers are shown in Table I.

Statistical analysis. Experimental data are presented as the mean \pm standard deviation. All the statistical analyses were performed using SPSS 19.0 statistical software (IBM Corp.) with one-way ANOVA followed by a Tukey's post hoc test. $\mathrm{P}<0.05$ was considered to indicate a statistically significant difference.

\section{Results}

Aberrant $m R N A$ expression profiles in $M C A O$ rats treated with PNS. Initially, no neurological deficit and infarction were observed in the Control and Sham groups. Compared with the Model group, it was found that PNS treatment attenuated the neurological deficit and infarction area (Fig. S1).
Additionally, aberrant mRNA expression profiles were analyzed between the Sham and Model groups, and between the Model and PNS-treated groups. According to the cut-off criteria 1,104 genes of interest were selected, where 690 genes were upregulated and 414 were downregulated in the Model group compared with the Sham group. Additionally, 817 genes of interest were selected, where 390 genes were upregulated and 427 were downregulated in the PNS-treated group compared with the Model group. Aberrant mRNA expression profiles between the Sham and Model groups, and between the Model and PNS-treated groups were identified using hierarchical cluster analysis of the data (Fig. 1). The 10 most upregulated and downregulated genes between the Sham and Model groups, and between the Model and PNS-treated groups are shown in Table II.

GO analysis and KEGG pathway analysis. GO functional enrichment analysis revealed that when comparing the Sham and Model groups, the upregulated genes involved 3,232 'biological processes', 477 'cellular components' and 830 'molecular functions'. The downregulated genes involved 3,240 'biological processes', 350 'cellular components' and 561 'molecular functions'. When comparing the Model and PNS-treated groups, GO functional enrichment analysis revealed that the upregulated genes involved 2,610 'biological processes', 352 'cellular components' and 445 'molecular functions'. The downregulated genes involved 3,963 'biological processes', 384 'cellular components' and 723 'molecular functions'. The top 10 significant 'biological process', 'cellular component' and 'molecular functions' identified by GO enrichment analysis for differentially expressed genes between the Sham and Model groups, and between the Model and PNS-treated groups are shown in Fig. 2. GO analysis suggested that genes upregulated following PNS treatment were primarily related to 'condensed chromosome', 'RAGE receptor binding' and 'meiotic chromosome segregation'. Downregulated genes after PNS treatment were mainly involved in 'presynapse', 'glutamate receptor binding', 'neuromuscular process'. KEGG analysis was 
Table II. Top 10 upregulated and downregulated aberrant mRNAs between the Sham group and Model group and between the Model group and PNS group.

A, Differentially expressed mRNAs between Sham and Model groups

\begin{tabular}{lr}
\hline Gene & $\log _{2}$ Fold \\
\hline Shox2 & 9.66 \\
LOC108348145 & 9.67 \\
Gns & 12.41 \\
Selenbp1 & 21.56 \\
Ppcdc & 21.71 \\
LOC100911572 & 21.74 \\
LOC100912537 & 22.64 \\
LOC103690164 & 22.72 \\
Gatd1 & 23.39 \\
LOC103692166 & 24.71 \\
Calcoco1 & -24.61 \\
LOC108348078 & -24.52 \\
Wbp11 & -23.43 \\
Rufy2 & -23.32 \\
Prmt6 & -22.25 \\
LOC100912585 & -21.77 \\
Map2k3 & -21.53 \\
AABR07035479.1 & -21.47 \\
Pidd1 & -21.38 \\
AABR07002870.2 & -21.20 \\
\hline
\end{tabular}

B, Differentially expressed mRNAs between Model and PNS groups

\begin{tabular}{lr}
\hline Gene & $\log _{2}$ Fold \\
\hline Calcoco1 & 24.66 \\
Rufy2 & 23.58 \\
Wbp11 & 23.39 \\
AC109542.1 & 21.64 \\
AABR07035479.1 & 20.63 \\
Cttn & 12.68 \\
Rs11d111 & 11.91 \\
LOC103690108 & 10.90 \\
Knop1 & 9.32 \\
Map2k3 & 9.10 \\
LOC100911572 & -21.78 \\
Is11 100910990 & -21.95 \\
LOC100 & -22.56 \\
LOC100912537 & -22.67 \\
LOC103690164 & -22.78 \\
Gatd1 & -23.43 \\
LOC100911881 & -23.91 \\
Cpne1 & -24.71 \\
LOC103692166 & -25.05 \\
LOC108348101 & -25.78 \\
\hline
\end{tabular}

PNS, Panax notoginseng saponin.
Table III. Expression change of the top 10 differentially expressed mRNAs based on MNC centrality.

\begin{tabular}{lcc}
\hline Gene & $\log _{2}$ Fold (Model/Sham) & $\log _{2}$ Fold (PNS/Model) \\
\hline UBE2V2 & -1.74 & 2.02 \\
SUMO1 & -1.14 & 1.19 \\
SSB & -1.81 & 1.92 \\
FAU & 1.18 & -1.18 \\
CEP290 & -1.10 & 1.13 \\
POLR2K & -1.09 & 1.08 \\
CUL4B & -1.25 & 1.03 \\
MATR3 & -1.37 & 1.09 \\
KDR & 1.20 & -1.52 \\
PRPF40A & -1.03 & 1.00
\end{tabular}

PNS, Panax notoginseng saponin; MNC, maximum neighborhood component; UBE2V2, ubiquitin conjugating enzyme E2 variant 2; SUMO1, small ubiquitin-related modifier 1; SSB, small RNA binding exonuclease protection factor La; FAU, Finkel-Biskis-Reilly murine sarcoma virus (FBR-MuSV) ubiquitously expressed; CEP290, centrosomal protein $290 \mathrm{kDa}$; POLR2K, DNA-directed RNA polymerase II subunit K; CUL4B, cullin-4B; MATR3, matrin-3; KDR, vascular endothelial growth factor receptor 2; PRPF40A, pre-mRNA-processing factor 40 homolog A.

performed to enrich the signaling pathways of differentially expressed genes between the Sham group and Model group or between the Model group and PNS-treated groups. KEGG analysis revealed that the upregulated genes between the Sham and Model groups were involved in 258 signaling pathways, whereas the downregulated genes between the Sham and Model groups were involved in 164 signaling pathways. Additionally, KEGG analysis revealed that the upregulated genes between the Model and PNS-treated groups were involved in 258 signaling pathways, whereas the downregulated genes between the Model and PNS-treated groups were involved in 226 signaling pathways. The top enrichment results are shown in Fig. 3. KEGG analysis indicated that upregulated genes after PNS treatment were primarily involved in the 'Herpes simplex virus 1 infection', 'Oocyte meiosis' and 'Cell cycle', whereas downregulated genes after PNS treatment were related to 'Glutamatergic synapses', 'Rap1 signaling pathway' and 'Calcium signaling pathway'.

PPI network analysis is important for understanding the interactions that occur between the proteins. The results of the PPI network analysis are shown in Fig. 4. Based on PPI network analysis, transmembrane protease, serine 2 (Tmprss2), dedicator of cytokinesis 8 (Dock8), 2'-5' oligoadenylate synthase (Oasl2), mannose receptor, C type 2 (Mrc2), glycoprotein (transmembrane) nmb (Gpnmb), lysosomal protein transmembrane 5 (Laptm5), colony stimulating factor 1 receptor (Csflr), transmembrane protein 176A (Tmem176a), AABR07035470.1 and WDFY family member $4(W d f y 4)$ were the top 10 differentially expressed genes between the Sham and Model groups. Furthermore, the top 10 differentially expressed genes between the Model group and PNS-treated groups were ubiquitin conjugating enzyme E2 variant 2 (UBE2V2), 
A
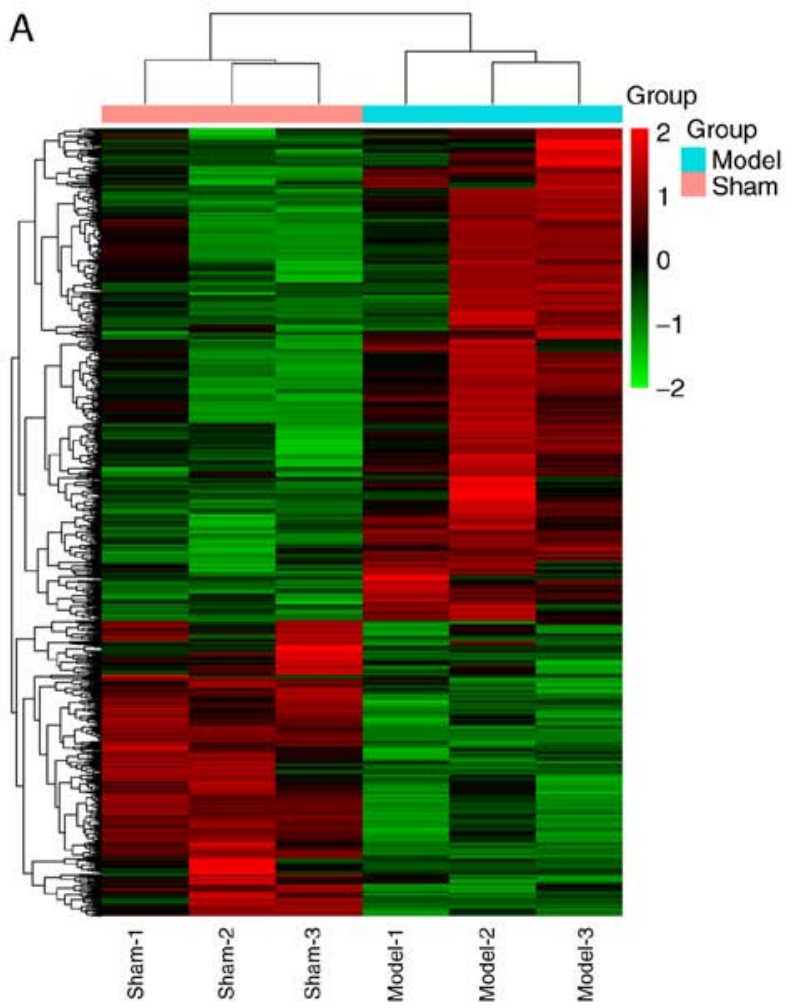

B

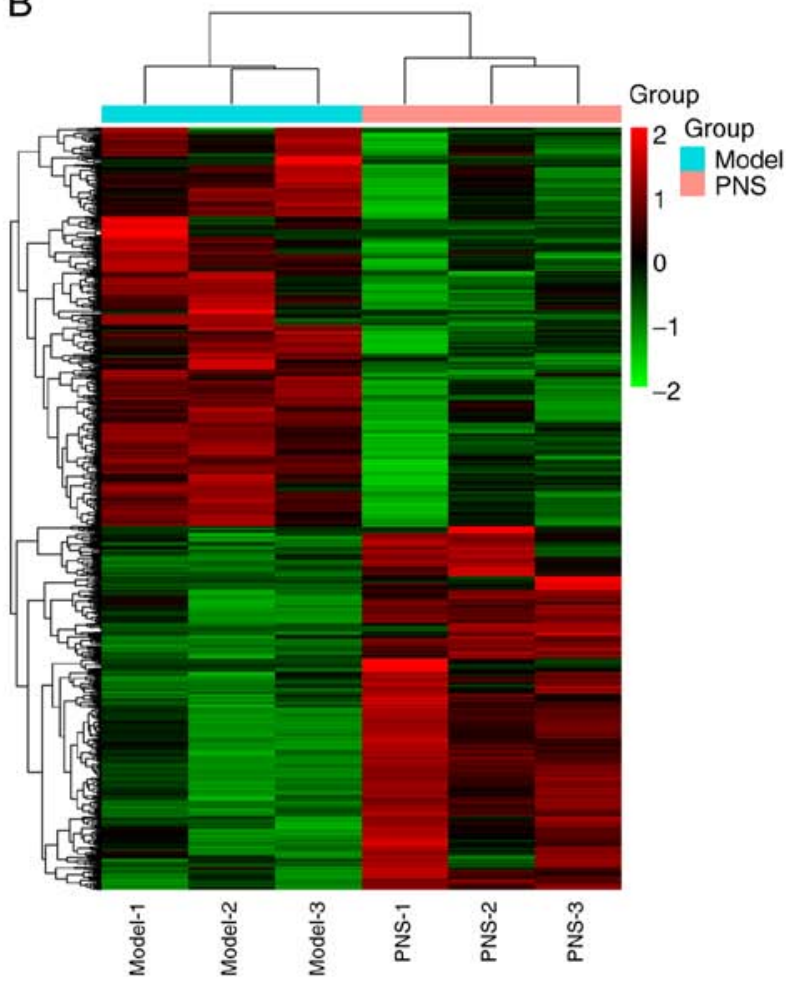

Figure 1. Heat map of aberrant mRNA expression profiles between (A) Sham and Model groups, and between the (B) Model and PNS-treated groups. Tissue samples are presented in vertical columns, while individual genes are presented in horizontal rows. Red indicates upregulated genes, and green indicates downregulated genes. PNS, Panax notoginseng saponin.

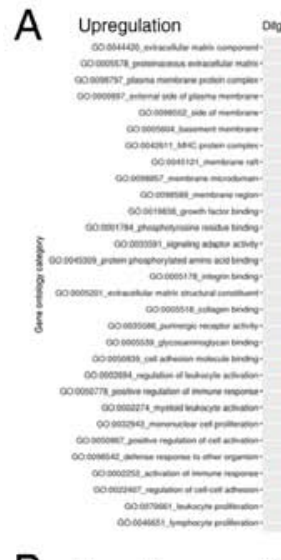

B Upregulation

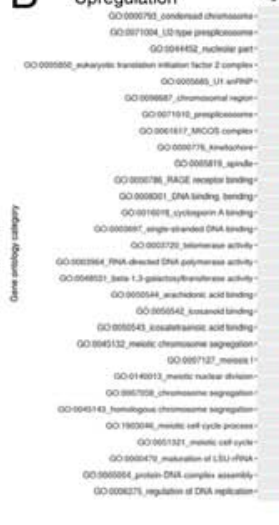

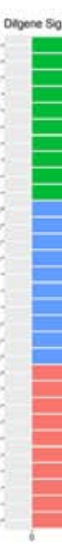

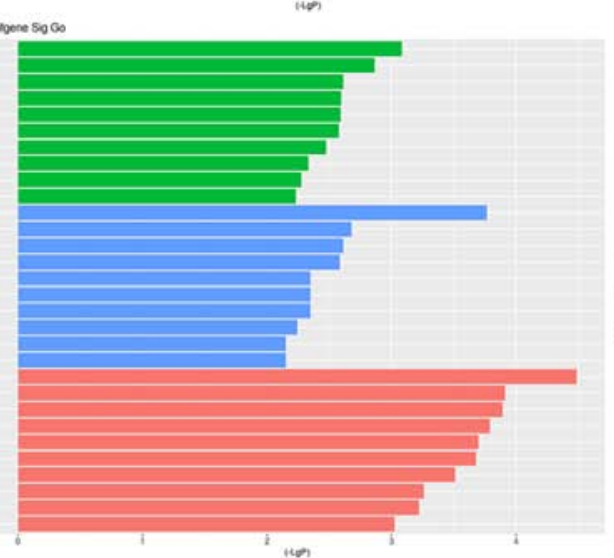

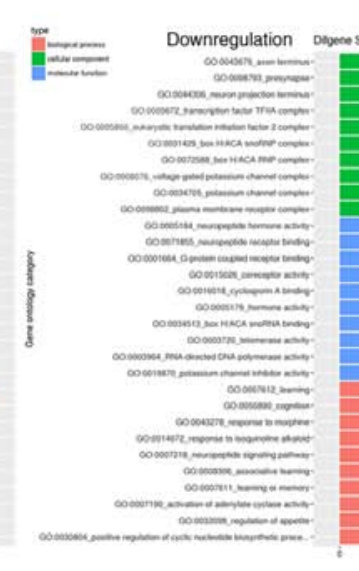
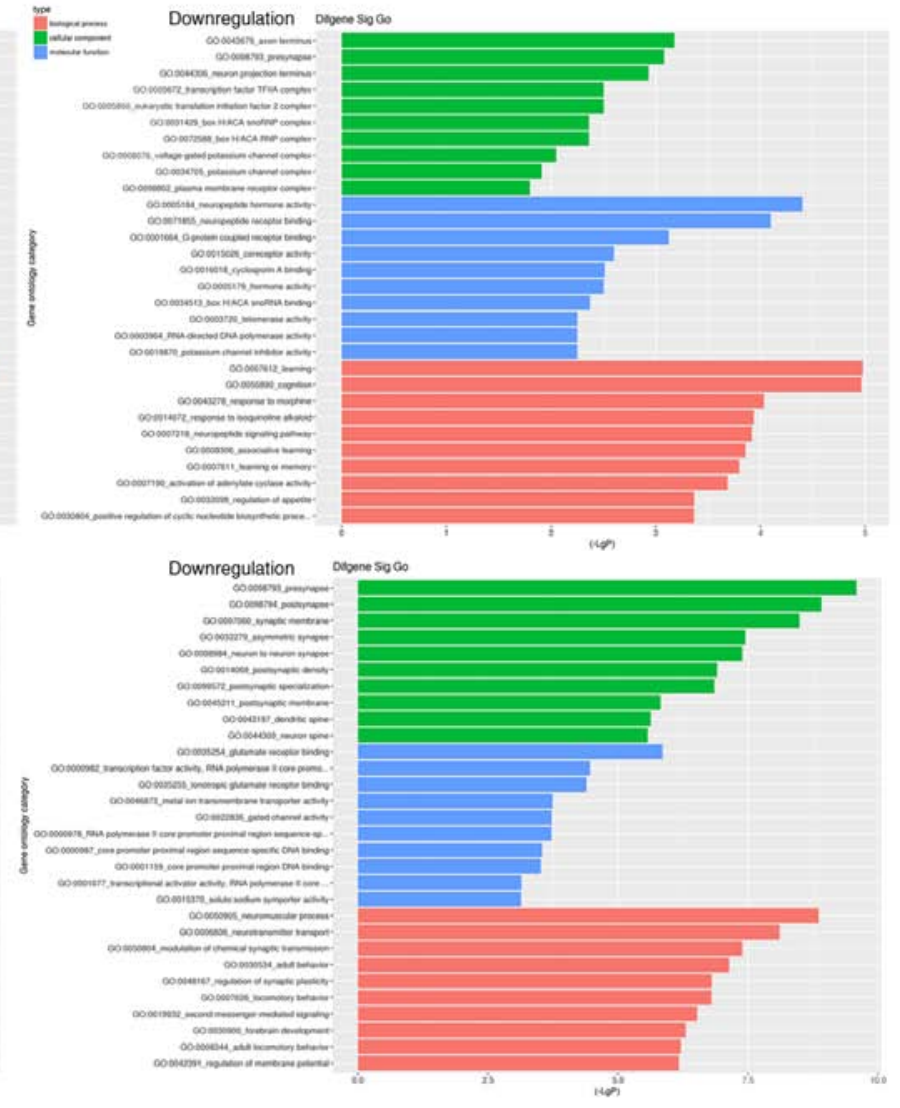

Figure 2. Top 10 most significant 'biological processes', 'cellular components' and 'molecular functions' determined by GO functional enrichment analysis for differentially expressed genes. (A) Comparison between the Sham group and Model group. (B) Comparison between the Model group and PNS-treated group. PNS, Panax notoginseng saponin; GO, Gene Ontology. 

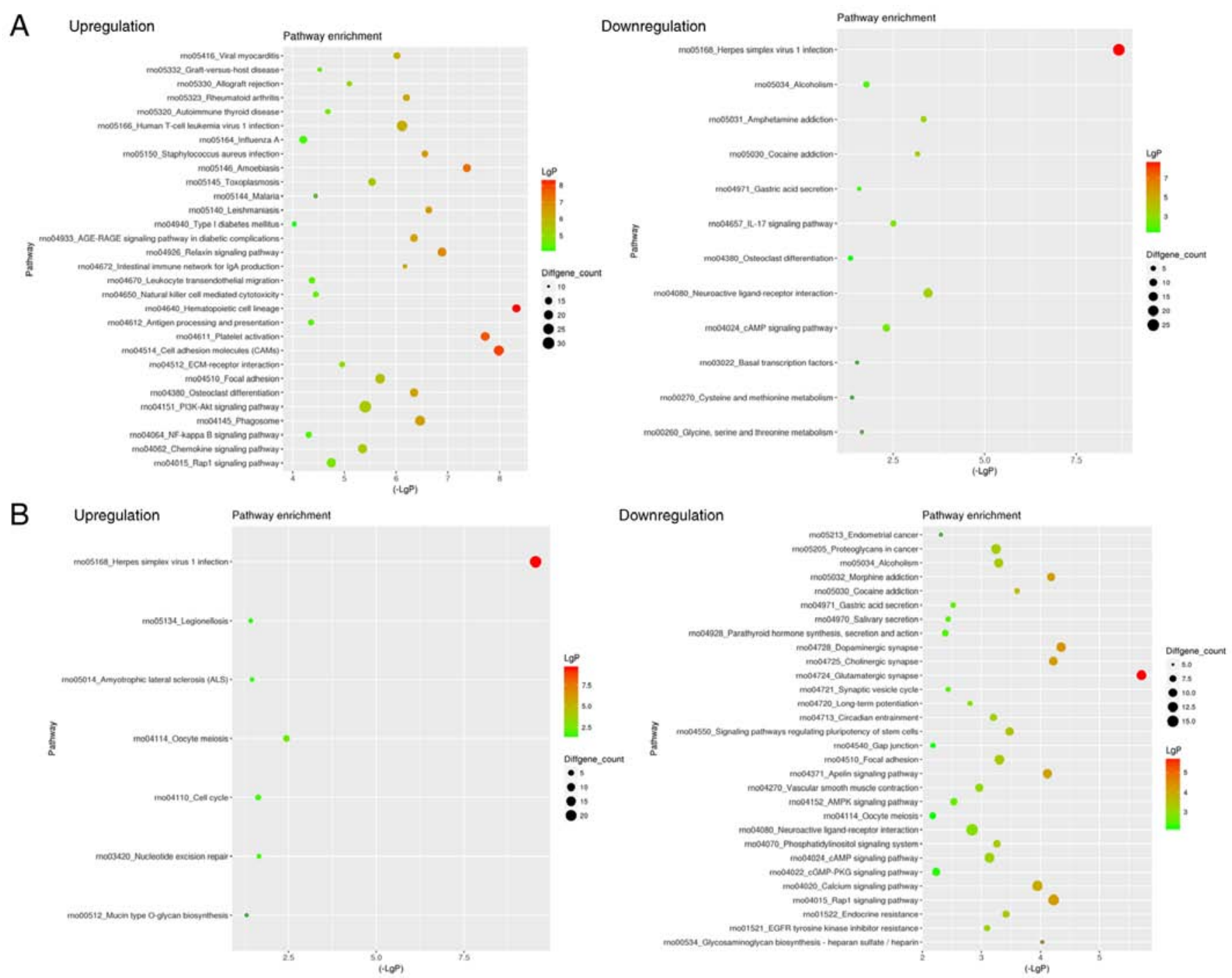

Figure 3. Significant pathways involving differentially expressed genes determined by Kyoto Encyclopedia of Genes and Genomes analysis. (A) Comparison between the Sham group and the Model group. (B) Comparison between the Model group and the Panax notoginseng saponin-treated group.

small RNA binding exonuclease protection factor La $(S S B)$, peptidylprolyl isomerase D (Ppid), CCHC-type zinc finger, nucleic acid binding protein $(C n b p)$, Zinc finger protein 788 (Zfp788), zinc finger protein 182 (Zfp182), AABR07035107.1, polyribonucleotide nucleotidyltransferase 1 (Pnptl), required for meiotic nuclear division 5 homolog A $($ Rmnd5a) and C1GALT1-specific chaperone 1 (Clgaltlcl).

Validating $m R N A$ expression by $R T-q P C R$. To analyze the aberrant expression of mRNAs that have important roles in ischemic stroke and the underlying mechanisms of PNS treatment, the overlapping genes of interest identified between Sham and Model groups, and between the Model and PNS-treated groups were analyzed using Venn diagrams, which demonstrated 303 overlapping genes (Fig. 5A). These 303 mRNAs were analyzed using STRING, and the hub genes were analyzed using CytoHubba to identify maximum neighborhood component (MNC) centrality in the Cytoscape software. Based on MNC centrality, the top 10 differentially expressed genes were identified as $U B E 2 V 2$, small ubiquitin-related modifier 1 (SUMOI), SSB, Finkel-Biskis-Reilly murine sarcoma virus ubiquitously expressed (FAU), centrosomal protein $290 \mathrm{kDa}(C E P 290)$, DNA-directed RNA polymerase II subunit K (POLR2K), cullin-4B (CUL4B), matrin-3 (MATR3), vascular endothelial growth factor receptor $2(K D R)$ and pre-mRNA-processing factor 40 homolog A (PRPF4OA) (Fig. 5B). Additionally, the present study also found that these top 10 genes were involved 'nucleotide excision repair', 'positive regulation of catabolic process', 'cell cycle' and 'regulation of cell cycle process' (Fig. 5C). The changes in expression in the top 10 genes are shown in Table III. The results showed that the expression levels of UBE2V2, SUMO1, SSB, CEP290, POLR2K, CUL4B, PRPF40A and MATR 3 were significantly reduced in the Model group compared with the Sham group, but was upregulated in the PNS-treated group. Expression of $F A U$ and $K D R$ was significantly increased in the Model group compared with the Sham group, but decreased in the PNS-treated group. Finally, the expression levels of $U B E 2 V 2$, SUMO1, SSB, FAU, CEP290, POLR2K, CUL4B, MATR3, PRPF40A and KDR were analyzed using RT-qPCR in the Control, Sham, Model, $50 \mathrm{mg} / \mathrm{kg}$ PNS, $100 \mathrm{mg} / \mathrm{kg}$ PNS and $150 \mathrm{mg} / \mathrm{kg}$ PNS treatment groups (Fig. 6). The expression levels of UBE2V2, SUMO1, $S S B, C E P 290, P O L R 2 K, C U L 4 B$, PRPF40A and MATR3 

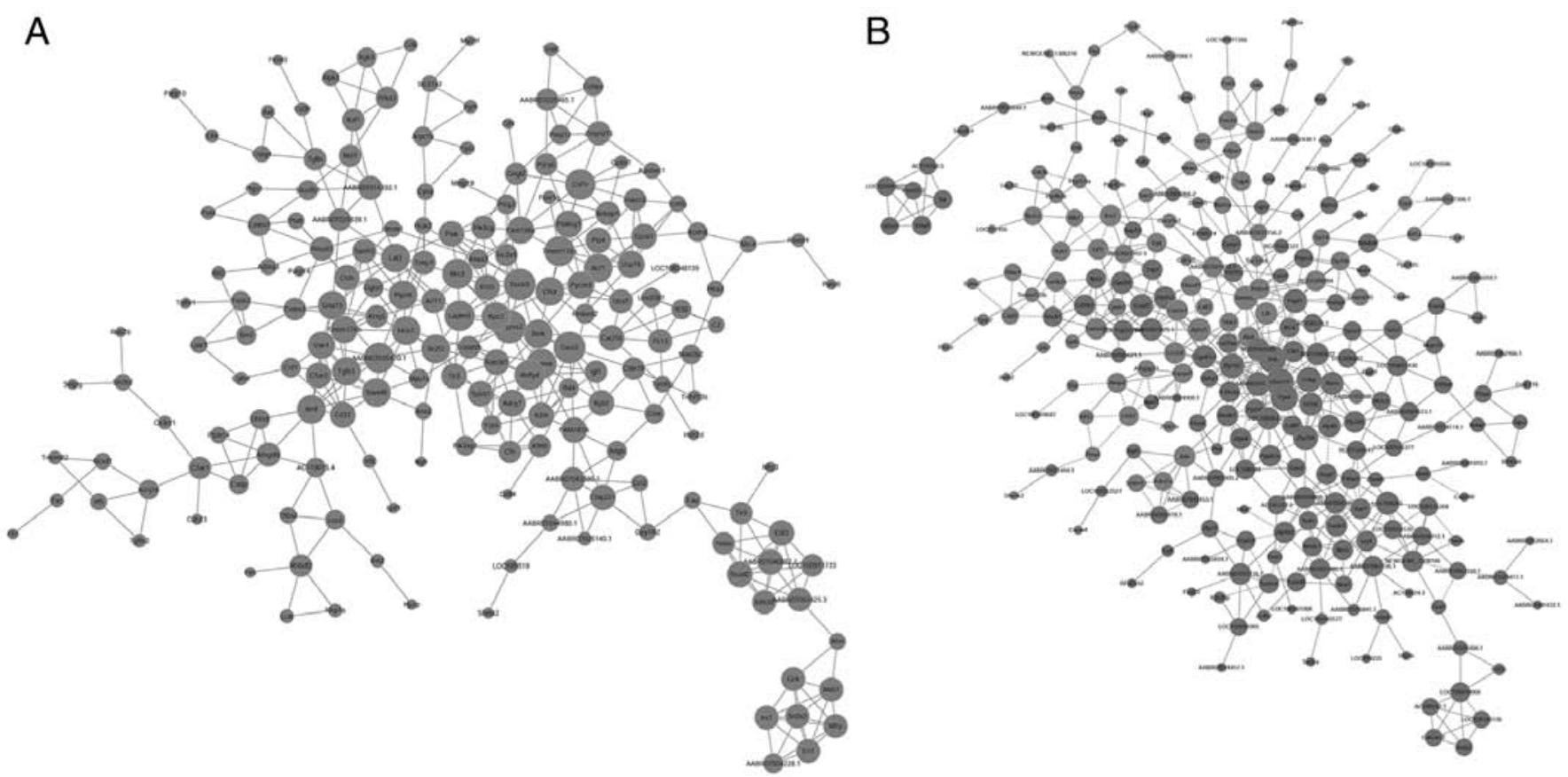

Figure 4. Protein-protein interactions network analysis was carried out using Search Tool for the Retrieval of Interacting Genes/Proteins. (A) Genes that were differentially expressed between the Sham group and the Model group. (B) Genes that were differentially expressed between the Model group and the Panax notoginseng saponin-treated group.

A

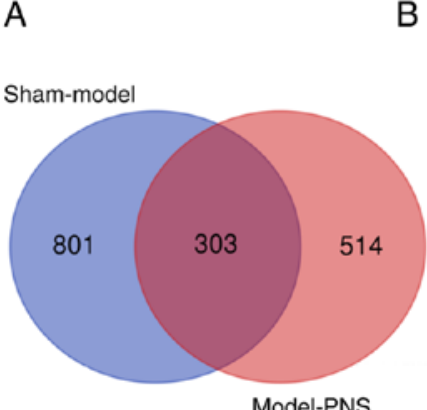

C

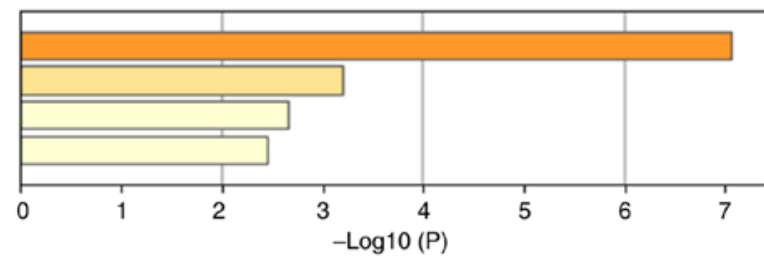

B

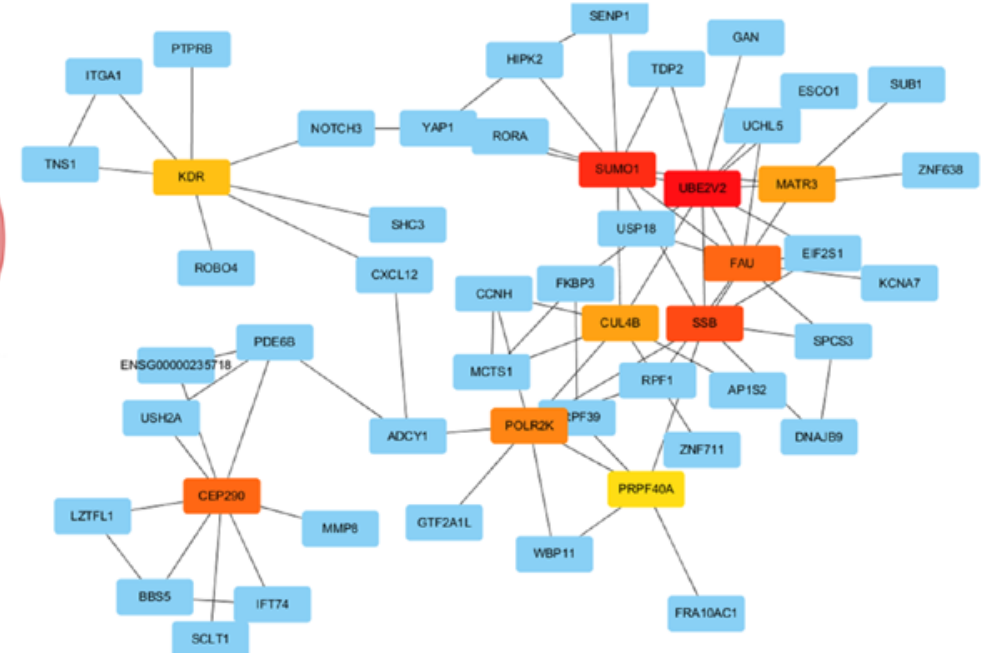

R-HSA-5696398: Nucleotide excision repair GO:0009896: Positive regulation of catabolic process R-HSA-1640170: Cell cycle GO:0010564: Regulation of cell cycle process

Figure 5. Top 10 hub genes from the 303 genes that were differentially expressed in both the Sham and Model group comparison, and the Model and PNS-treated group comparison. (A) A total of 303 overlapping genes that were aberrantly expressed between the Sham and Model groups and between the Model and PNS-treated groups were analyzed by Venn diagram analysis and selected from the overlap region of the Venn diagram. (B) All 303 genes were identified by CytoHubba (maximum neighborhood component centrality) in Cytoscape software, and the top 10 differentially expressed genes from the PPI network were selected. Colors indicate the number of PPIs: The number of PPIs in the order of highest to lowest is in red, orange, yellow and blue. Red-labeled proteins exhibited an increased number of PPIs compared with blue-labeled proteins. (C) The top 10 genes were primarily involved in 'nucleotide excision repair', 'positive regulation of catabolic process', 'cell cycle' and 'regulation of cell cycle process'. PNS, Panax notoginseng saponin; PPI, protein-protein interactions.

were significantly reduced, whereas $F A U$ and $K D R$ expression was significantly increased in the Model group compared with the Sham group. PNS treatment appeared to reverse the trends in gene expression regulation; the effects of 100 and 

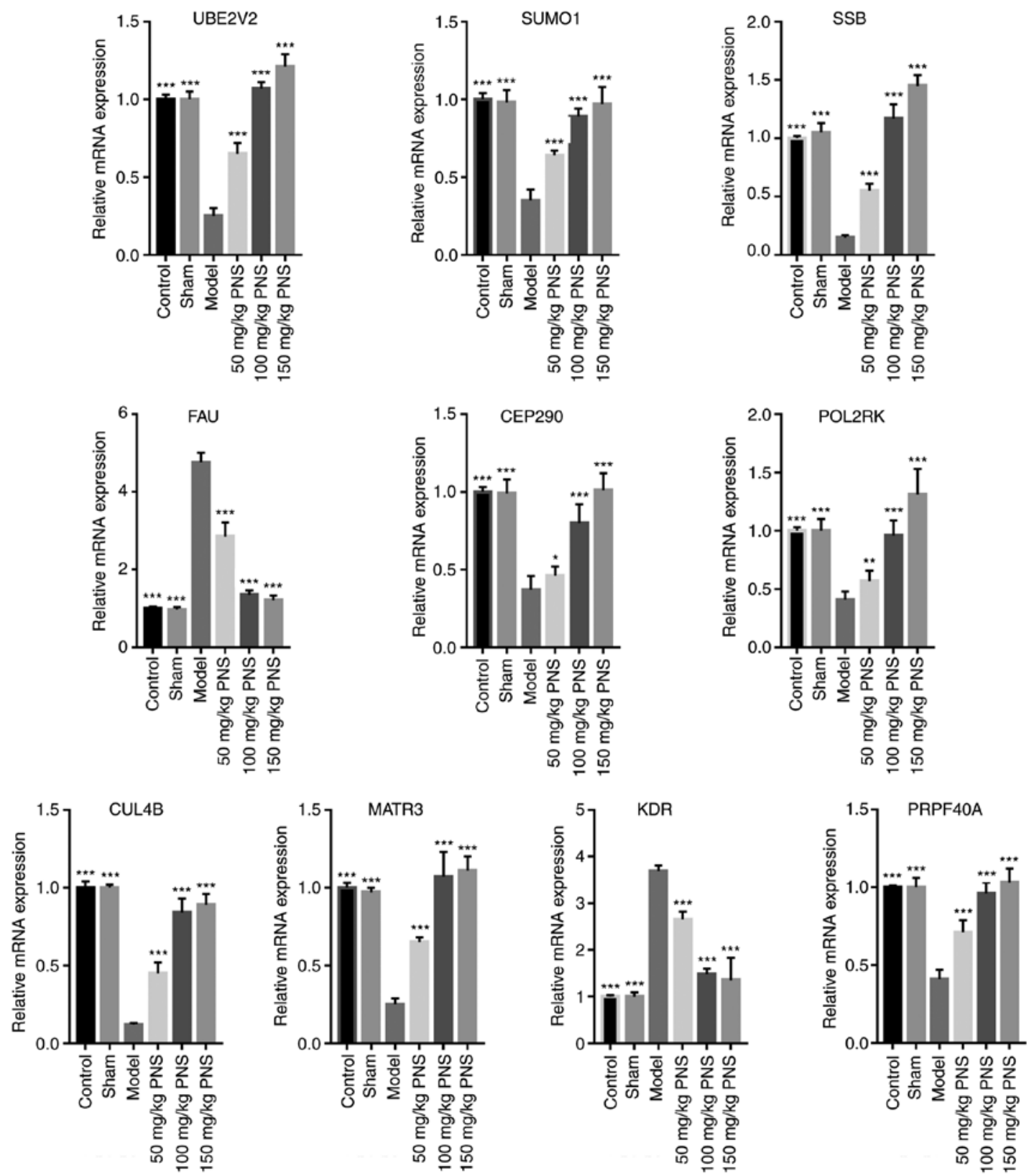

Figure 6. Expression of top 10 hub genes were validated by reverse transcription-quantitative PCR. $\mathrm{n}=10$, samples include the rats used for mRNA deep sequencing. ${ }^{*} \mathrm{P}<0.05,{ }^{* *} \mathrm{P}<0.01$ and ${ }^{* * * *} \mathrm{P}<0.001$ vs. Model group. PNS, Panax notoginseng saponin; UBE2V2, ubiquitin conjugating enzyme E2 variant 2 ; SUMO1, small ubiquitin-related modifier 1; SSB, small RNA binding exonuclease protection factor La; FAU, Finkel-Biskis-Reilly murine sarcoma virus ubiquitously expressed; CEP290, centrosomal protein 290 kDa; POLR2K, DNA-directed RNA polymerase II subunit K; CUL4B, cullin-4B; MATR3, matrin-3; KDR, vascular endothelial growth factor receptor 2; PRPF40A, pre-mRNA-processing factor 40 homolog A.

$150 \mathrm{mg} / \mathrm{kg}$ PNS treatment groups were more significant than that of the $50 \mathrm{mg} / \mathrm{kg}$ PNS treatment group.

\section{Discussion}

Various studies have demonstrated that PNS treatment can prevent neurological damage in MCAO model rats to promote recovery after a stroke $(8,16,17)$. The present study also found that PNS treatment attenuated the neurological deficit and infarction area. However, the exact molecular mechanisms are unclear and require further study. In the present study, mRNA sequencing was employed to investigate differential gene expression between the Model and Sham groups, and between the Model and PNS-treated groups. When comparing the
Model and Sham groups, 1,104 genes of interest were identified, including 690 upregulated and 414 downregulated genes. When the Model group was compared with the PNS-treated groups, 817 genes of interest were identified, which included 390 upregulated and 427 downregulated genes. These genes may be important in the underlying mechanisms of PNS in the treatment of ischemic stroke.

The genes identified via GO and KEGG analyses in the present study may indicate novel molecules or pathways underlying PNS treatment of ischemic stroke. Glutamatergic synapses and calcium signaling are known to play important roles in cerebral ischemia, contributing to neuronal death after a stroke $(29,30)$. Under ischemic stroke conditions, neuronal calcium signaling participates in endothelial restoration 
to stabilize intravascular thrombi (31). Rap1 is a member of the Ras family of small GTPases that activates the ERK pathway (32). Rap1 is also known to regulate cell-cell adhesion (33), promote angiogenesis and help maintain endothelial barrier functions in the event of a stroke $(34,35)$. The apelinergic system, which consists of apelin and apelin receptor, is temporally dysregulated during an ischemic stroke (36). Moreover, apelin signaling is known to reduce neuronal apoptosis and facilitate angiogenesis in post-stroke recovery (36). cAMP and phosphatidylinositol signaling pathways can attenuate cerebral ischemic injury by inhibiting neuronal apoptosis and stimulating angiogenesis (37-39). These results indicate that PNS attenuates ischemic stroke injury through a variety of signaling pathways. Wang et al (40) investigated the underlying mechanisms of Xuesaitong (XST; a Chinese medicine extracted from Panax notoginseng roots) in preventing stroke using microarray on a MCAO rat animal model, and found that the top differentially expressed pathways of XST against stroke included 'focal adhesion' and 'ECM-receptor interaction', which were also found in the present study.

Additionally, the present study found that 303 aberrantly expressed genes overlapped when comparing the Model and Sham groups, and the Model and PNS-treated groups. The top 10 differentially expressed genes among the 303 genes included UBE2V2, SUMO1, SSB, FAU, CEP290, POLR $2 K$, CUL4B, MATR3, PRPF4OA and KDR, which were identified using STRING and Cytoscape analysis. Additionally, the top 10 differentially expressed genes were involved in 'nucleotide excision repair', 'positive regulation of catabolic process', 'cell cycle' and 'regulation of cell cycle process'. Expression of UBE2V2, SUMO1, SSB, CEP290, POLR $2 \mathrm{~K}$, $C U L 4 B, P R P F 4 O A$ and MATR3 was significantly reduced in the Model group compared with the Sham group, whereas expression of $F A U$ and $K D R$ was significantly increased according to mRNA sequencing and RT-qPCR results. PNS treatment reversed these patterns of gene expression. UBE2V2 can reduce DNA damage responses, leading to worse neurological outcomes after stroke $(41,42)$. SUMO1 is known to have a neuroprotective role in ischemia/reperfusion injury by inhibiting neuron apoptosis (43). FAU is involved in apoptosis and metastasis in tumor progression and may affect the cell apoptosis in the nervous system following stroke (44). CEP290 knockout mice exhibit early vision loss, nephropathy and die from hydrocephalus (45). Hydrocephalus also occurs in the brain of patients with ischemic stroke, suggesting that CEP290 may be a target for the treatment of ischemic stroke. CUL4B can regulate Wnt/ $\beta$-catenin signaling, autophagy and Janus Kinase (JNK) signaling $(46,47)$. Wnt/ $\beta$-catenin signaling, autophagy and JNK signaling have important roles in oxidative stress, the breakdown of the blood-brain barrier and mitochondrial dysfunction in ischemic stroke (48-50). KDR is a surface marker of endothelial progenitor cells, and its expression is significantly reduced in patients with ischemic stroke $(51,52)$. These results showed that these genes of interest, including UBE2V2, SUMO1, FAU, CEP290 and CUL4B, are critical during PNS treatment of ischemic stroke. Additionally, $S S B$, POLR2K, MATR3, PRPF4OA and KDR are novel genes found in the present study that may have important effects in the development of ischemic stroke and in the PNS treatment of ischemic stroke. Wang et al (40) investigated the mechanism by which XST prevents strokes and found that fatty acid-binding protein 4 adipocyte $(F A B P 4)$, urokinase-type plasminogen activator ( $P L A U)$, heme oxygenase 1 (HMOXI), leukotriene $\mathrm{C} 4$ synthase (LTC4S), insulin-like growth factor I (Igfl) and secreted phosphoprotein 1 (SPPI) expression were increased by MCAO induction, and decreased by XST treatment (40). It was also found that FABP4, PLAU, $H M O X 1$ and $S P P 1$ expression were increased by MCAO and Igfl expression was inhibited by PNS treatment. The results indicated that there are some differences in the targets of PNS and XST in the treatment of stroke. The possible reasons are: i) composition of the two drugs may differ; ii) doses of the drugs used in the two studies are different; and iii) sequencing sample is too small, which may result in false negative results.

The present study has some limitations. Because the sequencing samples are small, a number of differentially expressed genes may not be identified effectively, alternatively, some genes may appear that are false positives. Additionally, the lack of a positive control to evaluate the therapeutic effect of PNS was also a limitation of the present study. Furthermore, the top 10 differentially expressed genes was only verified at the mRNA expression level, and western blotting and immunohistochemistry were not performed to investigate the protein expression of the target genes. Finally, at present there are no data to prove that these genes are altered in human patients who suffer from a stroke.

In conclusion, the present study found that 303 genes of interest overlapped between comparisons of the Model group and the Sham group, and between the Model group and the PNS-treated group. The 10 most notable hub genes among the 303 genes included UBE2V2, SUMO1, SSB, FAU, CEP290, POLR2K, CUL4B, MATR3 and KDR. These genes are potentially important during PNS treatment against ischemic stroke. The present findings provided novel insight into the pathogenesis of ischemic stroke.

\section{Acknowledgements}

Not applicable.

\section{Funding}

The present study was supported by the National Natural Science Foundation of China (grant no. 81460614).

\section{Availability of data and materials}

The datasets used and/or analyzed during the current study are available from the corresponding author on reasonable request.

\section{Authors' contributions}

JL, PL, LS and LM conceived and designed the present study, and developed the methodology. JL, PL, QH, CJ, JH, XT, XL and YL performed the experiments and collected the data. JL, XH, WH, PL and LM analyzed and interpreted the data. JL and PL drafted the manuscript. All authors read and approved the final manuscript. 


\section{Ethics approval and consent to participate}

The present study was approved by the Ethics Committee of Youjiang Medical College for National Institutional Review Board.

\section{Patient consent for publication}

Not applicable.

\section{Competing interests}

The authors declare that they have no competing interests.

\section{References}

1. Fuentes B and Tejedor ED: Stroke: The worldwide burden of stroke-a blurred photograph. Nat Rev Neurol 10: 127-128, 2014.

2. Moskowitz MA, Lo EH and Iadecola C: The science of stroke: mechanisms in search of treatments. Neuron 67: 181-198, 2010.

3. Duan J, Cui J, Yang Z, Guo C, Cao J, Xi M, Weng Y, Yin Y, Wang Y, Wei G, et al: Neuroprotective effect of Apelin 13 on ischemic stroke by activating AMPK/GSK-3 $\beta / \mathrm{Nrf} 2$ signaling. J Neuroinflammation 16: 24, 2019.

4. Bi BL, Wang HJ, Bian $\mathrm{H}$ and Tian ZT: Identification of therapeutic targets of ischemic stroke with DNA microarray. Eur Rev Med Pharmacol Sci 19: 4012-4019, 2015.

5. Wang J, Cao B, Han D, Sun M and Feng J: Long Non-coding RNA H19 induces cerebral ischemia reperfusion injury via activation of autophagy. Aging Dis 8: 71-84, 2017.

6. Liu N, Shan D, Li Y, Chen H, Gao Y and Huang Y: Panax notoginseng saponins attenuate phenotype switching of vascular smooth muscle cells induced by notch 3 silencing. Evid Based Complement Alternat Med 2015: 162145, 2015.

7. Li F, Zhao H, Han Z, Wang R, Tao Z, Fan Z, Zhang S, Li G, Chen $\mathrm{Z}$ and Luo Y: Xuesaitong may protect against ischemic stroke by modulating microglial phenotypes and inhibiting neuronal cell apoptosis via the STAT3 Signaling pathway. CNS Neurol Disord Drug Targets 18: 115-123, 2019.

8. Liu L, Zhu L, Zou Y, Liu W, Zhang X, Wei X, Hu B and Chen J: Panax notoginseng saponins promotes stroke recovery by influencing expression of Nogo-A, NgR and p75NGF, in vitro and in vivo. Biol Pharm Bull 37: 560-568, 2014.

9. Yang PF, Song XY and Chen NH: Advances in pharmacological studies of Panax notoginseng saponins on brain ischemia-reperfusion injury. Yao Xue Xue Bao 51: 1039-1046, 2016 (In Chinese)

10. Xie W, Meng X, Zhai Y, Zhou P, Ye T, Wang Z, Sun G and Sun X: Panax notoginseng saponins: A review of its mechanisms of antidepressant or anxiolytic effects and network analysis on phytochemistry and pharmacology. Molecules 23: 940, 2018.

11. Xu C, Wang W, Wang B, et al: Analytical methods and biological activities of Panax notoginseng saponins: Recent trends. J Ethnopharmacol 236: 443-465, 2019.

12. Shi $\mathrm{YH}, \mathrm{Li} \mathrm{Y}$, Wang $\mathrm{Y}, \mathrm{Xu} \mathrm{Z}, \mathrm{Fu} \mathrm{H}$ and Zheng GQ: Ginsenoside-Rb1 for ischemic stroke: A systematic review and meta-analysis of preclinical evidence and possible mechanisms. Front Pharmacol 11: 285, 2020.

13. Nabavi SF, Sureda A, Habtemariam S and Nabavi SM Ginsenoside $\mathrm{Rd}$ and ischemic stroke; a short review of literatures. J Ginseng Res 39: 299-303, 2015.

14. Xie CL, Wang WW, Xue XD, Zhang SF, Gan J and Liu ZG: A systematic review and meta-analysis of Ginsenoside-Rg1 (G-Rg1) in experimental ischemic stroke. Sci Rep 5: 7790, 2015.

15. Meng L, Lin J, Huang Q, Liang P, Huang J, Jian C, Lin C and Li X: Panax notoginseng saponins attenuate oxygen-glucose deprivation/reoxygenation-induced injury in human SH-SY5Y Cells by regulating the expression of inflammatory factors through miR-155. Biol Pharm Bull 42: 462-467, 2019.

16. Shi X, Yu W, Yang T, Liu W, Zhao Y, Sun Y, Chai L, Gao Y, Dong $\mathrm{B}$ and Zhu L: Panax notoginseng saponins provide neuroprotection by regulating NgR1/RhoA/ROCK2 pathway expression, in vitro and in vivo. J Ethnopharmacol 190: 301-312, 2016.
17. Shi X, Yu W, Liu L, Liu W, Zhang X, Yang T, Chai L, Lou L, Gao $\mathrm{Y}$ and Zhu L: Panax notoginseng saponins administration modulates pro-/anti-inflammatory factor expression and improves neurologic outcome following permanent MCAO in rats. Metab Brain Dis 32: 221-233, 2017.

18. Longa EZ, Weinstein PR, Carlson S and Cummins R: Reversible middle cerebral artery occlusion without craniectomy in rats. Stroke 20: 84-91, 1989.

19. Bederson JB, Pitts LH, Tsuji M, Nishimura MC, Davis RL and Bartkowski H: Rat middle cerebral artery occlusion: Evaluation of the model and development of a neurologic examination. Stroke 17: 472-476, 1986.

20. Ashburner M, Ball CA, Blake JA, Botstein D, Butler H, Cherry JM, Davis AP, Dolinski K, Dwight SS, Eppig JT, et al: Gene ontology: Tool for the unification of biology. The Gene Ontology Consortium. Nat Genet 25: 25-29, 2000.

21. The Gene Ontology Resource: 20 years and still GOing strong. Nucleic Acids Res 47: D330-D338, 2019.

22. Yu G, Wang LG, Han Y and He QY: ClusterProfiler: An $\mathrm{R}$ package for comparing biological themes among gene clusters. Omics 16: 284-287, 2012.

23. Kanehisa M and Goto S: KEGG: kyoto encyclopedia of genes and genomes. Nucleic Acids Res 28: 27-30, 2000.

24. Kanehisa M, Sato Y, Furumichi M, Morishima K and Tanabe M: New approach for understanding genome variations in KEGG. Nucleic Acids Res 47: D590-D595, 2019.

25. Kanehisa M: Toward understanding the origin and evolution of cellular organisms. Protein Sci 28: 1947-1951, 2019.

26. Szklarczyk D, Gable AL, Lyon D, Junge A, Wyder S, Huerta-Cepas J, Simonovic M, Doncheva NT, Morris JH, Bork P, et al: STRING v11: Protein-protein association networks with increased coverage, supporting functional discovery in genome-wide experimental datasets. Nucleic Acids Res 47: D607-D613, 2019.

27. Shannon P, Markiel A, Ozier O, Baliga NS, Wang JT, Ramage D, Amin N, Schwikowski B and Ideker T: Cytoscape: A software environment for integrated models of biomolecular interaction networks. Genome Res 13: 2498-2504, 2003.

28. Livak KJ and Schmittgen TD: Analysis of relative gene expression data using real-time quantitative PCR and the 2(-Delta Delta C(T)) method. Methods 25: 402-408, 2001.

29. Huang L, Wang C, Zhao S, Ge R, Guan S and Wang JH: PKC and CaMK-II inhibitions coordinately rescue ischemia-induced GABAergic neuron dysfunction. Oncotarget 8: 39309-39322, 2017.

30. Chen BH, Park JH, Lee YL, Kang IJ, Kim DW, Hwang IK, Lee CH, Yan BC, Kim YM, Lee TK, et al: Melatonin improves vascular cognitive impairment induced by ischemic stroke by remyelination via activation of ERK1/2 signaling and restoration of glutamatergic synapses in the gerbil hippocampus. Biomed Pharmacother 108: 687-697, 2018.

31. Secondo A, Bagetta G and Amantea D: On the role of store-operated calcium entry in acute and chronic neurodegenerative diseases. Front Mol Neurosci 11: 87, 2018.

32. Hattori M and Minato N: Rap1 GTPase: Functions, regulation, and malignancy. J Biochem 134: 479-484, 2003.

33. Kooistra MR, Dube N and Bos JL: Rap1: A key regulator in cell-cell junction formation. J Cell Sci 120: 17-22, 2007.

34. Chrzanowska-Wodnicka M: Rap1 in endothelial biology. Curr Opin Hematol 24: 248-255, 2017.

35. Chrzanowska-Wodnicka M: Distinct functions for Rap1 signaling in vascular morphogenesis and dysfunction. Exp Cell Res 319: 2350-2359, 2013.

36. Wu Y, Wang X, Zhou X, Cheng B, Li G and Bai B: Temporal expression of apelin/apelin receptor in ischemic stroke and its therapeutic potential. Front Mol Neurosci 10: 1, 2017.

37. Hu S, Cao Q, Xu P, Ji W, Wang G and Zhang Y: Rolipram stimulates angiogenesis and attenuates neuronal apoptosis through the cAMP/cAMP-responsive element binding protein pathway following ischemic stroke in rats. Exp Ther Med 11: 1005-1010, 2016.

38. Bai H, Zhao L, Liu H, Guo H, Guo W, Zheng L, Liu X, Wu X, Luo J, Li X, et al: Adiponectin confers neuroprotection against cerebral ischemia-reperfusion injury through activating the cAMP/PKA-CREB-BDNF signaling. Brain Res Bull 143: 145-154, 2018. 
39. Kim YS, Yoo A, Son JW, Kim HY, Lee YJ, Hwang S, Lee KY, Lee YJ, Ayata C, Kim HH and Koh SH: Early activation of phosphatidylinositol 3-kinase after ischemic stroke reduces infarct volume and improves long-term behavior. Mol Neurobiol 54: 5375-5384, 2017

40. Wang L, Yu Y, Yang J, Zhao X and Li Z: Dissecting Xuesaitong's mechanisms on preventing stroke based on the microarray and connectivity map. Mol Biosyst 11: 3033-3039, 2015.

41. Zhao Y, Long MJC, Wang Y, Zhang S and Aye Y: Ube2V2 Is a rosetta stone bridging redox and ubiquitin codes, coordinating DNA damage responses. ACS Cent Sci 4: 246-259, 2018.

42. Li P, Stetler RA, Leak RK, Shi Y, Li Y, Yu W, Bennett MVL and Chen J: Oxidative stress and DNA damage after cerebral ischemia: Potential therapeutic targets to repair the genome and improve stroke recovery. Neuropharmacology 134: 208-217, 2018

43. Zhang H, Wang Y, Zhu A, Huang D, Deng S, Cheng J, Zhu MX and Li Y: SUMO-specific protease 1 protects neurons from apoptotic death during transient brain ischemia/reperfusion. Cell Death Dis 7: e2484, 2016

44. Perina D, Korolija M, Hadzija MP, Grbeša I, Belužić R, Imešek M Morrow C, Marjanović MP, Bakran-Petricioli T, Mikoč A and Ćetković H: Functional and structural characterization of FAU gene/protein from marine sponge suberites domuncula. Mar Drugs 13: 4179-4196, 2015.

45. Rachel RA, Yamamoto EA, Dewanjee MK, May-Simera HL, Sergeev YV, Hackett AN, Pohida K, Munasinghe J, Gotoh N, Wickstead B, et al: CEP290 alleles in mice disrupt tissue-specific cilia biogenesis and recapitulate features of syndromic ciliopathies. Hum Mol Genet 24: 3775-3791, 2015.

46. He YM, Xiao YS, Wei L, Zhang JQ and Peng CH: CUL4B promotes metastasis and proliferation in pancreatic cancer cells by inducing epithelial-mesenchymal transition via the $\mathrm{Wnt} / \beta$-catenin signaling pathway. J Cell Biochem 119: 5308-5323, 2018.
47. Li Y, Zhou X, Zhang Y, Yang J, Xu Y, Zhao Y and Wang X: CUL4B regulates autophagy via JNK signaling in diffuse large B-cell lymphoma. Cell Cycle 18: 379-394, 2019.

48. Jean LeBlanc N, Menet R, Picard K, Parent G, Tremblay ME and ElAli A: Canonical Wnt pathway maintains blood-brain barrier integrity upon ischemic stroke and its activation ameliorates tissue plasminogen activator therapy. Mol Neurobiol 56: 6521-6538, 2019

49. Wang P, Shao BZ, Deng Z, Chen S, Yue Z and Miao CY: Autophagy in ischemic stroke. Prog Neurobiol 163-164: 98-117, 2018.

50. Zheng J, Dai Q, Han K, Hong W, Jia D, Mo Y, Lv Y, Tang H, Fu H and Geng W: JNK-IN-8, a c-Jun N-terminal kinase inhibitor, improves functional recovery through suppressing neuroinflammation in ischemic stroke. J Cell Physiol 235: 2792-2799, 2019.

51. Deng Y, Wang J, He G, Qu F and Zheng M: Mobilization of endothelial progenitor cell in patients with acute ischemic stroke. Neurol Sci 39: 437-443, 2018

52. Marti-Fabregas J, Delgado-Mederos R, Crespo J, Peña E, Marín R, Jiménez-Xarrié E, Fernández-Arcos A, Pérez-Pérez J, Martínez-Domeño A, Camps-Renom P, et al: Circulating endothelial progenitor cells and the risk of vascular events after ischemic stroke. PLoS One 10: e0124895, 2015.

This work is licensed under a Creative Commons Attribution-NonCommercial-NoDerivatives 4.0 International (CC BY-NC-ND 4.0) License. 